\title{
Origin of the Extended Van Hove region in Cuprate Superconductors
}

\author{
Wei-Guo Yin, $, 1,2, *$ Chang-De Gong, ${ }^{3,1,2}$ and P. W. Leung ${ }^{4}$ \\ ${ }^{1}$ Department of Physics, Nanjing University, Nanjing 210093, People's Republic of China \\ ${ }^{2}$ National Key Laboratory of Solid States of Microstructure, Nanjing University, Nanjing 210093, China \\ ${ }^{3}$ CCAST (World Laboratory), P.O. Box 8730, Beijing 100080, China \\ ${ }^{4}$ Department of Physics, Hong Kong University of Science and Technology, Clear Water Bay, Hong Kong
}

(Received 16 October 1997; revised manuscript received 3 April 1998)

\begin{abstract}
We study the evolution of the quasiparticle dispersion with doping for the two-dimensional $t-t^{\prime}-t^{\prime \prime}-J$ model using the exact diagonalization technique and present a consistent theoretical analysis. Both calculations show that the flat region around $(\pi, 0)$ does not exist at half filling, however, it appears in the underdoped regime and remains almost unchanged upon additional doping. The enhancement of the incoherent motion of holes induced especially by the $t^{\prime}$ and $t^{\prime \prime}$ terms accounts for the formation of the extended Van Hove region. Our results are in excellent agreement with the recent photoemission experiments on $\mathrm{Bi}_{2} \mathrm{Sr}_{2} \mathrm{Ca}_{1-x} \mathrm{Dy}_{x} \mathrm{Cu}_{2} \mathrm{O}_{8+\delta}$ [Marshall et al., Phys. Rev. Lett. 76, 4841 (1996)]. [S0031-9007(98)07129-4]

PACS numbers: 74.25.Jb, 71.27.+a, 74.20.Mn
\end{abstract}

One of the most intriguing advances in cuprate superconductivity is the recent observation of an extended Van Hove singularity (EVHS) near the Fermi surface in the angle-resolved photoemission spectroscopy (ARPES) of optimally doped Y-Ba-Cu-O [1] and $\mathrm{Bi}_{2} \mathrm{Sr}_{2} \mathrm{CaCu}_{2} \mathrm{O}_{8+\delta}$ [2] that is extensively considered in the studies of many other anomalous properties of the cuprate superconductors $[3,4]$. The origin of the EVHS is thus of crucial importance in the search for the microscopic theory of hightemperature superconductivity.

Theoretically, the flat region around $(\pi, 0)$ was found [5] in the single-hole dispersion of the two-dimensional (2D) $t-J$ model by using the self-consistent Born approximation (SCBA) to treat the scattering of a single hole with the spin waves of the Néel state [6]. Many numerical studies supported this result $[7,8]$, and indicated that the quasiparticle dispersion of the $t-J$ model is approximately independent of doping $[9,10]$. On this basis, Dagotto et al. suggested that the EVHS is a consequence of the antiferromagnetic (AF) order [3]. However, recent photoemission experiments on differently doped $\mathrm{Bi}_{2} \mathrm{Sr}_{2} \mathrm{Ca}_{1-x} \mathrm{Dy}_{x} \mathrm{Cu}_{2} \mathrm{O}_{8+\delta}$ [11] reported a strong dopingdependent quasiparticle band structure: the flat region around $(\pi, 0)$ still remains in the underdoped regime at an energy of $200 \mathrm{meV}$ below the Fermi surface, but disappears in the undoped regime where the photoemission spectra are similar to those from the $\mathrm{AF}$ insulator $\mathrm{Sr}_{2} \mathrm{CuO}_{2} \mathrm{Cl}_{2}$ (Ref. [12]) [i.e., the band energy at $(\pi, 0)$ is $0.3 \mathrm{eV}$ below the top of the valence band at $(\pi / 2, \pi / 2)]$. These observations doubt the adequacy of the $t$ - $J$ model for the description of cuprate superconductors. The origin of the experimentally observed EVHS is still unclear.

The purpose of this Letter is to show that the 2D $t-t^{\prime}-t^{\prime \prime}-J$ model is a better starting point. In the literature, the model was introduced by Nazarenko et al. to reproduce the quasiparticle dispersion of insulating $\mathrm{Sr}_{2} \mathrm{CuO}_{2} \mathrm{Cl}_{2}$ [13-15]. Whether it is fit for the cuprate superconductors is a current issue of physical interest $[16,17]$. In this Letter, we calculate the doping dependence of the photoemission spectra for the model using the numerical exact diagonalization technique (ED) and propose a theoretical analysis in spirit similar to that of Dagotto et al., where the underdoped regime is presented as a gas of spin polarons. Our numerics unambiguously show that the evolution of the quasiparticle dispersion with doping is in excellent agreement with the ARPES data [11]. The photoemission spectra in the whole momentum space look more incoherent in the underdoped regime than in the undoped regime. Consistently, our analytical calculations indicate that the enhancement of the incoherent motion of holes, which is induced especially by the $t^{\prime}$ and $t^{\prime \prime}$ terms, accounts for the formation of the EVHS. The antiferromagnetically induced spinpolaron picture provides indeed a natural description of the physics of the cuprates and a consistent way of interpreting the numerical and experimental results.

The Hamiltonian considered here is

$$
\begin{aligned}
H= & J \sum_{\langle i j\rangle_{1}} \mathbf{S}_{i} \cdot \mathbf{S}_{j} \\
& -\left(t \sum_{\langle i j\rangle_{1}, \sigma}+t^{\prime} \sum_{\langle i j\rangle_{2}, \sigma}+t^{\prime \prime} \sum_{\langle i j\rangle_{3}, \sigma}\right) \tilde{c}_{i \sigma}^{\dagger} \tilde{c}_{j \sigma},
\end{aligned}
$$

in the standard notation of the constrained Fermi operators. The angle brackets denote the first $\left(\langle i j\rangle_{1}\right)$, second $\left(\langle i j\rangle_{2}\right)$, and third $\left(\langle i j\rangle_{3}\right)$ neighbor sites, respectively. The model parameters are fixed: $t \simeq 0.4 \mathrm{eV}, J / t \simeq 0.4$, $t^{\prime} / t \simeq-0.3$, and $t^{\prime \prime} / t \simeq 0.2$. They are fits to the band structures of $\mathrm{Bi}_{2} \mathrm{Sr}_{2} \mathrm{CaCu}_{2} \mathrm{O}_{8+\delta}$ and $\mathrm{YBa}_{2} \mathrm{Cu}_{3} \mathrm{O}_{7-\delta}$ [18].

The photoemission spectrum (PES) is defined by

$$
A(\mathbf{k}, \omega)=\sum_{\nu}\left|\left\langle\nu_{n+1}\left|\tilde{c}_{\mathbf{k}, \sigma}\right| 0_{n}\right\rangle\right|^{2} \delta\left(\omega-E_{0}^{n}+E_{\nu}^{n+1}\right),
$$


where $E_{0}^{n}$ and $0_{n}$ are the ground-state energy and the wave function of the model with $n$ holes, and $E_{\nu}^{n+1}$ and $\nu_{n+1}$ are the energy and wave function of the $v$ th eigenstate with $n+1$ holes. $A(\mathbf{k}, \omega)$ is calculated using the standard Lanczos algorithm [19] with 500 iterations and an artificial broadening factor $\eta=0.05 t$. The calculations are performed in the standard 16- and 18-site clusters. The results from these clusters agree with each other.

Figure 1(a) shows the PES of the 0-hole (half filling) and 2-hole ground states of the 16-site cluster. At half filling, there are three main features in the spectral functions: (i) The lowest energy state of the hole quasiparticle locates at $(\pi / 2, \pi / 2)$ and the state at $(\pi, 0)$ moves considerably to higher energy comparable with the bandwidth; (ii) the spectral weights near $(\pi, 0)$ become quite weak and result in the difficultly resolved quasiparticle peaks in the ARPES experiments; (iii) the energy difference between the lowest PES peak at $(\pi, 0)$ and that at $(\pi / 2,0)$ is $\sim 0.4 t$. Coming to the 2-hole doped case, the PES reveals substantial changes of the band structure. The peaks near $(\pi, 0)$ are shifted towards lower binding energy, and the spectral weights near $(\pi, 0)$ increase simultaneously. Moreover, the lowest PES peak at $(\pi, 0)$

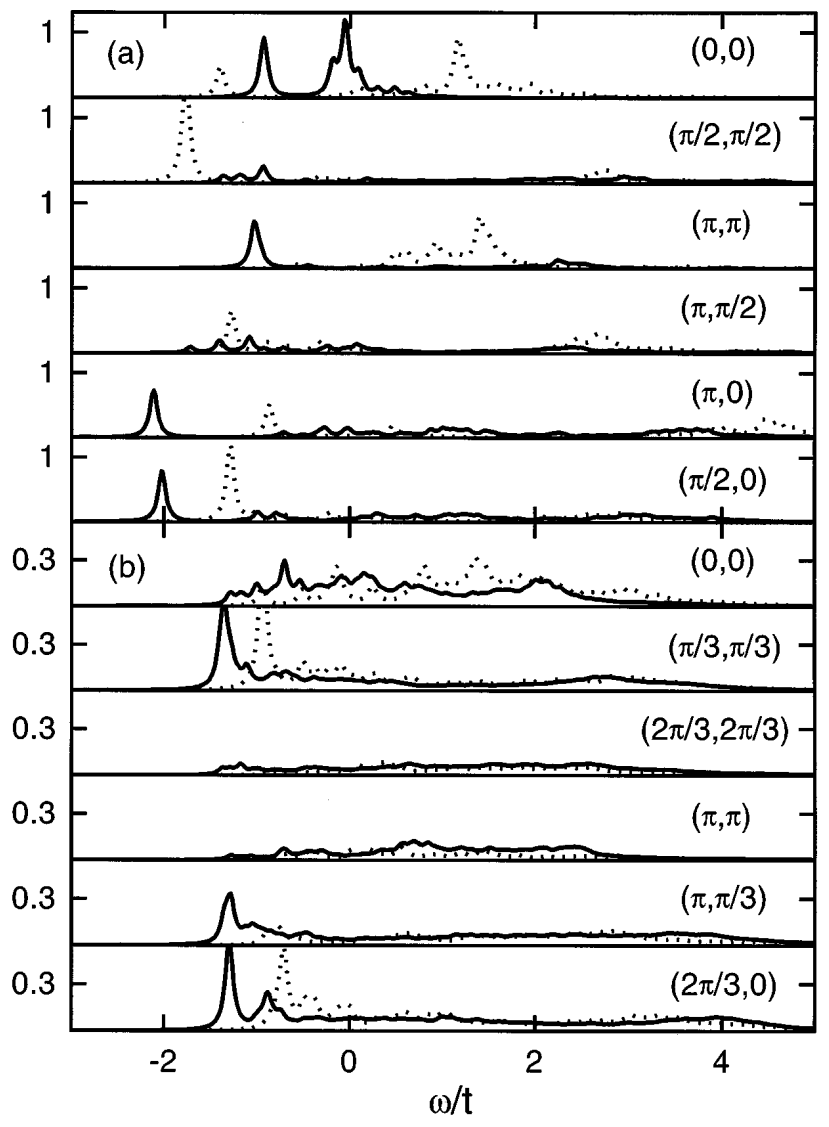

FIG. 1. The PES for the $t-t^{\prime}-t^{\prime \prime}-J$ model with (a) 0 hole (dotted line) and 2 holes (solid line) for a 16-site cluster; (b) 2 holes (solid line) and 4 holes (dotted line) for an 18 -site cluster. stands at almost the same energy as that at $(\pi / 2,0)$. This feature remains in the 4-hole ground state and is considered as the signal of the presence of the flat region. The quasiparticle dispersion of the 16-site cluster is illustrated in Fig. 2. Notice that we have shifted the ground-state energy at different doping to the same level (zero here), since the chemical potential is difficult to tell for such a small cluster. We believe that the shift will not change the physics concerned. The consistent results are obtained from the 18-site cluster as shown in Fig. 1(b): The PES of the 4-hole ground state is similar to the PES of the 2-hole ground state in the whole momentum space, except for a slight energy shift of the shape lines. These numerics indicate that the quasiparticle dispersion evolves with doping in two different ways: first in an evidently nonrigid way until the flat region appears and then in an approximately rigid way upon doping.

To understand the physics of the doping induced band deformation, we perform an analytical calculation of the quasiparticle dispersion. The main assumption is that as long as the $\mathrm{AF}$ correlation length $\xi_{\mathrm{AF}}$ is at least two lattice spacings, resembling the NMR experimental situation in $\mathrm{YBa}_{2} \mathrm{Cu}_{3} \mathrm{O}_{6.94}$ [20], the spin-polaron picture $[3,6,21]$ found at half filling survives a finite hole density. In the normal state of the cuprate superconductors at low-hole doping, the direct interaction between holes is neglected. Many-hole effects are contained in the doping induced modification of the underlying spin correlations. In the formal perturbation theory, the first-order matrix element of the kinetic energy is given by [22]

$$
\begin{aligned}
\mathbf{I}_{i j} & =\sum_{\sigma}\left\langle\tilde{c}_{i, \uparrow}^{\dagger} \tilde{c}_{i, \sigma} \tilde{c}_{j, \sigma}^{\dagger} \tilde{c}_{j, \uparrow}\right\rangle \\
& =\frac{1}{4}+\left\langle\mathbf{S}_{i} \cdot \mathbf{S}_{j}\right\rangle+\frac{1}{2}\left(\left\langle S_{i}^{z}\right\rangle+\left\langle S_{j}^{z}\right\rangle\right) .
\end{aligned}
$$

Note that $\mathbf{I}_{i j}=0(1)$ for the $t\left(t^{\prime}, t^{\prime \prime}\right)$ term in the Néel state. This means that the $t^{\prime}$ and $t^{\prime \prime}$ terms contribute precisely to the coherent motion of holes since transferring a hole to a

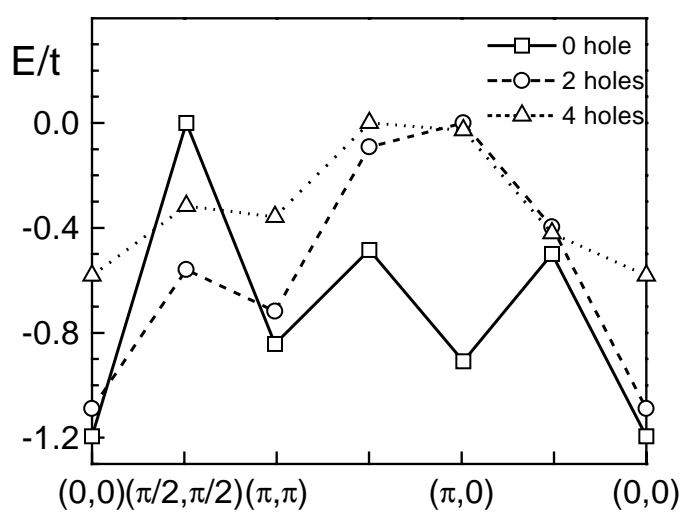

FIG. 2. The ED results of the quasiparticle dispersion for the $t-t^{\prime}-t^{\prime \prime}-J$ model in the 16 -site cluster. Note that we report the electron spectrum which is related to the hole spectrum in Fig. 1 by the transformations $\mathbf{k} \rightarrow \mathbf{k}+(\pi, \pi)$ and $\omega \rightarrow-\omega$. 
second- or third-nearest neighbor does not distort the AF background, while the $t$ term connects the hole motion only with the emission of the spin excitations. However, if the neighboring spins are not completely antiparallel, i.e., they have nonzero overlap, the doped holes can hop coherently onto the opposite sublattice and result in the experimentally observed and numerically obtained asymmetry of the quasiparticle dispersion between $k$ and $k+Q$, where $Q=(\pi, \pi)[12,14,21,23]$. Meanwhile, the spins on the same sublattice no longer align perfectly along the same direction; as a result, the $t^{\prime}$ and $t^{\prime \prime}$ terms can enhance the emission of the spin excitations, thus contributing to the hole-spin-wave coupling function $M_{k q}$. To mimic this, in our previous paper we have analytically derived an effective spin-polaron Hamiltonian of the form [21]

$$
H_{\mathrm{eff}}=\sum_{q} \omega_{q} b_{q}^{\dagger} b_{q}+\sum_{k} \epsilon_{k} h_{k}^{\dagger} h_{k}+\sum_{k q} h_{k}^{\dagger} h_{k-q} b_{q} M_{k q},
$$

where $h$ and $b$, are the spinless fermion and magnon operators, respectively. $\omega_{q}=2 J \sqrt{1-\gamma_{q}^{2}}$ is the linear spin-wave spectrum. The bare hole dispersion has the form [21]

$$
\epsilon_{k}=4 C_{1} t \gamma_{k}+4 C_{2} t^{\prime} \lambda_{k}+4 C_{3} t^{\prime \prime} \eta_{k},
$$

where the renormalization factors $C_{1}, C_{2}$, and $C_{3}$ are $\mathbf{I}_{i j}$ for $\langle i j\rangle_{1},\langle i j\rangle_{2}$, and $\langle i j\rangle_{3}$, respectively. $\gamma_{k}=\frac{1}{2}\left(\cos k_{x}+\right.$ $\left.\cos k_{y}\right), \quad \lambda_{k}=\cos k_{x} \cos k_{y}, \quad$ and $\quad \eta_{k}=\frac{1}{2}\left(\cos 2 k_{x}+\right.$ $\cos 2 k_{y}$ ). The hole-spin-wave coupling function is [21]

$$
\begin{aligned}
M_{k q}= & \frac{4}{\sqrt{N}}\left[u_{q}\left(t \gamma_{k-q}+t^{\prime} \lambda_{k-q}+t^{\prime \prime} \eta_{k-q}\right)\right. \\
& \left.+v_{q}\left(t \gamma_{k}+t^{\prime} \lambda_{k}+t^{\prime \prime} \eta_{k}\right)\right],
\end{aligned}
$$

where $u_{q}, v_{q}$ are the Bogolubov transformation parameters of the linear spin waves [6].

Figure 3(a) shows our analytical results using the SCBA [6] in a $16 \times 16$ square lattice. The values of the spinspin correlation function in Eq. (3) are phenomenologically replaced by our ED results for the $t-t^{\prime}-t^{\prime \prime}-J$ model (see Table I). Note that, at half filling, $\left\langle S_{i}^{z}\right\rangle \sim 0.35$; at finite doping, $\left\langle S_{i}^{z}\right\rangle=0$. The calculated quasiparticle dispersion is in excellent agreement with the experimental results (Fig. 3 in Ref. [11]) and the numerical results (Fig. 2). As shown in Table I, $C_{2}$ and $C_{3}$ are much reduced as the AF order is depleted, thus decreasing the influence of the $t^{\prime}$ and $t^{\prime \prime}$ terms on the bare hole dispersion. Consequently, the band energy at $(\pi, 0)$ moves with respect to the Fermi energy from large to small as the hole density grows. Nevertheless, the $t^{\prime}$ and $t^{\prime \prime}$ terms in Eq. (5) cannot be renormalized towards zero even at high doping since the minimal value of $C_{2}$ or $C_{3}$ is 0.25 . At this point, if the $t^{\prime}$ and $t^{\prime \prime}$ terms contributed only to the coherent motion of holes (i.e., not included in the hole-spin-wave coupling function, $M_{k q}$ ), the flat region would no longer exist, as shown in Fig. 3(b). Therefore, the $t^{\prime}$ and $t^{\prime \prime}$ terms

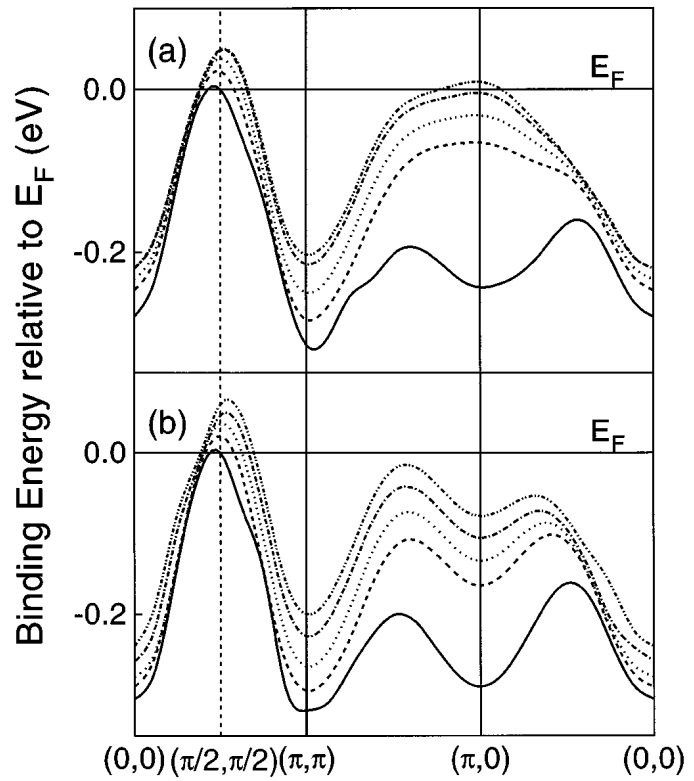

FIG. 3. (a) The quasiparticle dispersion for the $t-t^{\prime}-t^{\prime \prime}-J$ model in a $16 \times 16$ square lattice. The hole concentration $x$, from bottom to top at the $(\pi, 0)$ point, is $0,0.06,0.11,0.17$, and 0.22 , as listed in Table I. (b) Same as (a) but with the $t^{\prime}$ and $t^{\prime \prime}$ terms not included in $M_{k q}$.

weaken the spin correlation, enhance the incoherent motion of holes, and lead to the formation of the EVHS in the cuprate superconductors. This is consistent with the ED situation (Fig. 1), where the PES looks more incoherent in the underdoped regime than in the undoped regime.

Another interesting result in Fig. 3(a) is the rigidity of the EVHS about $(\pi, 0)$. That is, once the flat region has already formed, it tends to stay there upon additional doping. This can be intuitively understood according to the above interpretation since higher doping will further weaken the spin correlations. It also gives a natural interpretation as to why the quasiparticle band of the $t-J$ model is so rigid. In Fig. 3(a), the flat region appears at the small hole concentration $x \sim 0.06$, and the quasiparticle band from $x \sim 0.11$ (the underdoped regime) to 0.22 (near optimal doping [24]) remains almost unchanged. This feature (i.e., approximate rigidity of the quasiparticle dispersion over such a wide range of doping

TABLE I. The ED results of the spin-spin correlation function, $\left\langle\mathbf{S}_{i} \cdot \mathbf{S}_{j}\right\rangle$, for the $t-t^{\prime}-t^{\prime \prime}-J$ model in an 18-site cluster at different hole concentrations $x$.

\begin{tabular}{lccc}
\hline \hline$x$ & $\langle i j\rangle_{1}$ & $\langle i j\rangle_{2}$ & $\langle i j\rangle_{3}$ \\
\hline 0 & -0.347 & 0.217 & 0.200 \\
0.06 & -0.267 & 0.133 & 0.098 \\
0.11 & -0.209 & 0.093 & 0.046 \\
0.17 & -0.152 & 0.048 & 0.001 \\
0.22 & -0.118 & -0.013 & -0.019 \\
0.28 & -0.061 & -0.011 & -0.014 \\
\hline \hline
\end{tabular}


from underdoped to optimally doped) implies that many theoretical predictions based on the $t-J$ model survive in the $t-t^{\prime}-t^{\prime \prime}-J$ model.

In summary, we calculate the photoemission spectra for the differently hole doped 2D $t-t^{\prime}-t^{\prime \prime}-J$ model by using the numerical and analytical approaches, and show that the experimentally observed two-step evolution of the quasiparticle dispersion with doping is reproduced in this basic model. The formation of the EVHS is a consequence of the enhancement of the incoherent motion of holes induced especially by the $t^{\prime}$ and $t^{\prime \prime}$ terms.

We are grateful to E. Dagotto, A. Nazarenko, H. Q. Lin, J.X. Li, M. R. Li, and Y.J. Wang for their helpful discussions and suggestions. This research was supported by the National Natural Science Foundation of China and the Research Grants Council of Hong Kong.

*Current address: Physics Department, Fudan University, Shanghai 200433, China.

Electronic address: wgyin@fudan.edu.cn

[1] K. Gofron et al., Phys. Rev. Lett. 73, 3302 (1994); J. Phys. Chem. Solids 54, 1193 (1993).

[2] D. S. Dessau et al., Phys. Rev. Lett. 71, 2781 (1993).

[3] E. Dagotto, A. Nazarenko, and A. Moreo, Phys. Rev. Lett. 74, 310 (1995).

[4] D. M. Newns, C.C. Tsuei, and P. C. Pattnaik, Phys. Rev. B 52, 13611 (1995).

[5] G. Martinez and P. Horsch, Phys. Rev. B 44, 317 (1991); Z. Liu and E. Manousakis, ibid. 45, 2425 (1992).
[6] S. Schmitt-Rink, C. M. Varma, and A.E. Ruckenstein, Phys. Rev. Lett. 60, 2793 (1988).

[7] P. W. Leung and R. J. Gooding, Phys. Rev. B 52, R15 711 (1995).

[8] E. Dagotto, A. Nazarenko, and M. Boninsegni, Phys. Rev. Lett. 73, 728 (1994).

[9] S. Haas, A. Moreo, and E. Dagotto, Phys. Rev. Lett. 74, 4281 (1995); A. Moreo, S. Haas, A. W. Sandvik, and E. Dagotto, Phys. Rev. B 51, 12045 (1995).

[10] R. Preuss, W. Hanke, and W. von der Linden, Phys. Rev. Lett. 75, 1344 (1995).

[11] D. S. Marshall et al., Phys. Rev. Lett. 76, 4841 (1996).

[12] B. O. Wells et al., Phys. Rev. Lett. 74, 964 (1995).

[13] A. Nazarenko et al., Phys. Rev. B 51, 8676 (1995).

[14] P. W. Leung, B. O. Wells, and R. J. Gooding, Phys. Rev. B 56, 6320 (1997).

[15] W. G. Yin and C. D. Gong, Phys. Rev. B 56, 2843 (1997); B. Kyung and R. Ferrell, ibid. 54, 10125 (1996).

[16] D. Duffy et al., Phys. Rev. B 56, 5597 (1997).

[17] R. Eder, Y. Ohta, and G. A. Sawatzky, Phys. Rev. B 55, R3414 (1997).

[18] O. K. Andersen et al., Phys. Rev. B 49, 4145 (1994); J. Phys. Chem. Solids 56, 1573 (1995).

[19] E. Dagotto, Rev. Mod. Phys. 66, 763 (1994).

[20] T. Imai et al., Phys. Rev. B 47, 9158 (1993).

[21] W. G. Yin, B. Hao, and C. D. Gong, Phys. Lett. A 220, 281 (1996).

[22] E. Dagotto et al., Phys. Rev. B 41, 9049 (1990).

[23] T. K. Lee and C. T. Shih, Phys. Rev. B 55, 5983 (1997).

[24] The hole density of optimally doped $\mathrm{Bi}_{2} \mathrm{Sr}_{2} \mathrm{CaCu}_{2} \mathrm{O}_{8+\delta}$ and $\mathrm{YBa}_{2} \mathrm{Cu}_{3} \mathrm{O}_{7-\delta}$ is about 0.25 ; see L.F. Feiner, J.H. Jefferson, and R. Raimondi, Phys. Rev. Lett. 76, 4939 (1996). 